\title{
All-trans retinoic acid upregulates the expression of p53 via Axin and inhibits the proliferation of glioma cells
}

\author{
JIANRONG LU ${ }^{1,2}$, FENG ZHANG $^{2}$, YONG YUAN ${ }^{1}$, CAIXIA DING $^{1}$, LIYING ZHANG $^{2}$ and QING LI $^{2}$ \\ ${ }^{1}$ Department of Pathology, Shaanxi Province Cancer Hospital, Xi'an, Shaanxi 710061; \\ ${ }^{2}$ State Key Laboratory of Cancer Biology, Department of Pathology, Xijing Hospital, \\ The Fourth Military Medical University, Xi'an, Shaanxi 710032, P.R. China
}

Received January 21, 2013; Accepted March 11, 2013

DOI: $10.3892 /$ or.2013.2391

\begin{abstract}
All-trans retinoic acid (ATRA) is a potent chemopreventive and therapeutic agent and exerts its effects by inducing growth arrest. In the present study, we demonstrated that ATRA activated the expression of p53 via Axin and induced cell cycle arrest at the G1/S phase and apoptosis of glioma cells. Briefly, C6 cells were treated with ATRA, and the levels of p53 mRNA and protein were determined by RT-PCR, western blotting and immunohistochemistry. The results showed that ATRA activated the expression of p53. In addition, ectopic expression of Axin by transient transfection of C6 cells with rAxin revealed that overexpression of Axin induced cell cycle arrest and apoptosis with an upregulation of p53. Furthermore, loss-of-function of Axin in glioma cells by RNAi blocked ATRA-induced cell cycle phase arrest and apoptosis via downregulation of p53. The present study revealed a novel function of Axin and identified it as an important regulator of ATRA-activated p53 expression.
\end{abstract}

\section{Introduction}

All-trans retinoic acid (ATRA) is a member of the retinoid family which includes substances structurally or funtionally related to retinol (vitamin A). ATRA exerts a profound effect on the survival, growth and differentiation of many types of cells during prenatal and adult life (1). Since 1989, when ATRA was introduced as a targeted therapy against acute promyelocytic leukemia (APL), it has been used extensively as an antitumor agent for many types of tumors (2). Several molecular mechanisms of ATRA have been identified in the induction of tumors undergoing cell cycle arrest and apoptosis by activating RAR and RXR (3-5). ATRA regulates the fate of cells also by other pathways, including p53 (6-10). These

Correspondence to: Professor Qing Li, Department of Pathology, Xijing Hospital, The Fourth Military Medical University, Xi'an, Shaanxi 710032, P.R. China

E-mail: liqing@fmmu.edu.cn

Key words: all-trans retinoic acid, proliferation, apoptosis, Axin, cell cycle arrest, glioma cell line, p53 studies revealed that ATRA induces cell cycle arrest and apoptosis by regulating the transcriptional activity of p53 and/ or stability of its protein. However, it remains unclear whether p53 take part in ATRA-induced cell death in glioma cells.

Axin is a negative regulator of axis formation in the development of mouse embryos. Its deficiency leads to axis duplication (11). Overexpression of Axin blocked embryo axis formation in Xenopus and caused apoptosis in transgenic mice and certain cell lines $(12,13)$. Accumulating data show that Axin emerges as a major scaffold for many pathways, including Wnt signaling, JNK mitogen-activated protein kinase signaling and transforming growth factor (TGF) $\beta$ signaling (14-16). Notably, a previous study revealed that Axin participates in ATRA-induced differentiation of embryonic carcinoma cells (17), suggesting that Axin plays an important role in retinoid-mediated cell fate. Furthermore, we previously found that ATRA activates the transcription of Axin in glioma cell lines (18). Studies have demonstrated that Axin forms a complex with p53 and induces p53-mediated apoptosis by stimulating p53 transcriptional activity $(19,20)$.

In the present study, the expression of p53 was found to be highly upregulated by ATRA treatment in the glioma C6 cell line. Ectopic expression of Axin induced cell cycle arrest and apoptosis and activated the expression of p53. Furthermore, the knockdown of Axin blocked ATRA-induced cell cycle arrest and apoptosis with downregulation of p53. These findings indicate that ATRA activated the intrinsic upregulation of p53 by a novel mechanism involved in the activation of Axin.

\section{Materials and methods}

Cell cultures and transfection. The rat C6 glioma cell line was cultured in Dulbecco's modified Eagle's medium (DMEM) (Invitrogen) supplemented with $10 \%$ (vol/vol) heat-inactivated fetal bovine serum in a humidified atmosphere with $5 \% \mathrm{CO}_{2}$. The glioma C6 cells were transfected with pIRES2-EGFPAxin and pIRES2-EGFP using Lipofectamine ${ }^{\mathrm{TM}} 2000$ reagent (Invitrogen) according to the manufacturer's protocol. After an 8-h transfection, cells were cultured with DMEM for $24 \mathrm{~h}$.

Growth curve assay. For the growth curve assay (MTT methods), C6 cells were plated in a 96-well plate at a density 
of $1 \times 10^{3}$ cells/well, and incubated in a humidified $5 \% \mathrm{CO}_{2}$ at $37^{\circ} \mathrm{C}$ and transfected with Axin siRNAs for $8 \mathrm{~h}$. After 24,48 and $72 \mathrm{~h}$, methyl thiazolyl tetrazolium (MTT) $(20 \mu \mathrm{l} /$ well) was added to the cells. After a 4-h incubation, the supernatants were removed, and $150 \mu \mathrm{l}$ dimethyl sulfoxide (DMSO) was added to each well and swirled for $10 \mathrm{~min}$ to solubilize the crystals. The absorbance was measured at $490 \mathrm{~nm}$.

Analysis of apoptosis. TUNEL method was used for analysis of apoptosis using an apoptosis detection kit (Maixin, China) according to the manufacturer's protocol. Briefly, cell slices were washed with PBS and digested with proteinase $\mathrm{K}$ for $3 \mathrm{~min}$. Then, slices were labeled with TdT and DIG-d-UTP for $2 \mathrm{~h}$ at $37^{\circ} \mathrm{C}$, and blocked with the blocking reagents at room temperature for $30 \mathrm{~min}$. The anti-Dig-antibody was applied to the cells and incubated at $37^{\circ} \mathrm{C}$ for $30 \mathrm{~min}$. After washing with PBS, slices were incubated with SABC at $37^{\circ} \mathrm{C}$ for $1 \mathrm{~h}$, and visualized with $\mathrm{DAB}$, and counterstained with hematoxylin. The apoptotic cells in five different fields were counted, and the apoptotic index was calculated as the ratio of apoptotic cells over total cells.

Flow cytometric analysis. Cells $\left(1 \times 10^{6}\right)$ were washed twice with PBS and pelleted at 1,000 x g for $5 \mathrm{~min}$. Cells were trypsinized and fixed with $70 \%$ ethanol and washed with PBS and resuspended in PBS containing RNAase $\mathrm{A}$ and propidium iodide (PI) in the dark for $10 \mathrm{~min}$ at $4^{\circ} \mathrm{C}$. The DNA contents of the stained nuclei were analyzed on a Becton-Dickinson FACScan flow cytometer. The distribution of DNA content categorized cells as being in the G1 and S phases. The cells with DNA content less than G1 were categorized in pre-G1 (hypodiploid cells) and determined as apoptotic phase cells.

Immunohistochemical staining. The cultured cells were incubated with 5\% normal goat serum (Sigma) and incubated overnight at $4^{\circ} \mathrm{C}$ with a mouse monoclonal antibody for $\mathrm{p} 53$ (Sigma). Following washing with PBS, cells were incubated at room temperature with biotinylated goat anti-mouse/rabbit IgG followed by streptavidin enzyme conjugate (Zhongshan, China) at room temperature. The reaction product was visualized by diaminobenzidine tetrahydrochloride (DAB). All sections were counterstained with hematoxylin. Quantification of the percentage of immunoreactive cells was determined by capturing images from random fields.

RNA interference. RNA interference (RNAi) was employed in this study to inhibit Axin expression and analyze the effect of Axin on ATRA-inhibited cell growth and the expression of p53. Duplex RNAi oligos with a two-nucleotide overhang at the end of the sequence were designed and synthesized at GenePharm Co. The sequence of RNA interference was as follows (Axin1-2253): sense sequence, 5'-GUAUCGUUGUG GCCUACUATT-3' and antisense sequence, 5'-UAGUAGGCC ACAACGAUACTG-3'. The negative control siRNA has no known specific effects on gene expression: sense sequence, 5'-UUCUCCGAACGUGUCACGUTT-3' and antisense sequence, 5'-ACGUGACACGUUCGGAGAATT-3'. C6 cells were transfected with siRNAs at a final concentration of $100 \mathrm{nM}$ using Lipofectamine 2000 reagent according to the manufacturer's instructions. At $8 \mathrm{~h}$ post-transfection, cells were cultured by administration of $2.5 \mu \mathrm{M}$ of ATRA for $24 \mathrm{~h}$. The interference efficiency exceeded $60 \%$ by comparison with the negative control.

$R T-P C R$. Total RNA was isolated using TRIzol (Invitrogen) according to the manufacturer's instructions. Single-stranded cDNA was synthesized from $1 \mu \mathrm{g}$ of total RNA using the PrimeScript $^{\mathrm{TM}}$ RT reagent kit following the protocol recommended by the manufacturer (Takara). PCR primers were as follows: Axin forward, 5'-AGGGTCTGGAACAGGGAA-3' and reverse, 5'-GGATAGCGTGTCAGCATCA-3'; p53 forward, 5'-GCGTTGCTCTGATGGTGA-3' and reverse, 5'-CAGCGTGATGATGGTAAGGA-3'; $\beta$-actin forward, 5'-TC ACCCACACTGTGCCCATCTA-3' and reverse, 5'-CATC GGAACCGCTCATTGCCGATAG-3'. A template-free negative control was included in each experiment. The PCR cycle was preceded by an initial denaturation of $5 \mathrm{~min}$ at $94^{\circ} \mathrm{C}$ followed by a final extension of $10 \mathrm{~min}$ at $72^{\circ} \mathrm{C}$. Each PCR regime was followed by 30 cycles at $94^{\circ} \mathrm{C}$ for $30 \mathrm{sec}, 58^{\circ} \mathrm{C}$ for $30 \mathrm{sec}$, and $72^{\circ} \mathrm{C}$ for $45 \mathrm{sec}$. Band intensity was quantified by Bandscan software. The gray values were expressed in relation to that of the control and presented as means \pm SD of three independent experiments.

Western blot analysis. For western blot analysis, cells were lysed in 2X SDS loading buffer. Total extracts were obtained in the supernatant. Approximately $20 \mu 1$ of the samples was resolved on $10 \%$ SDS-PAGE, and transferred to PVDF membranes. For western blot analysis a mouse monoclonal antibody for p53 (Sigma), a mouse polyclonal antibody for Axin (Santa Cruz Biotechnology) and a rabbit polyclonal antibody for $\beta$-actin (Sigma) were used to detect the corresponding proteins. Peroxidase-conjugated goat anti-mouse/ rabbit secondary antibody (Sigma) was used, and then the proteins were detected using an enhanced chemiluminescence reagent (Pierce). Band intensity was quantified by Bandscan software. Protein expression was normalized by the quantity of $\beta$-actin and presented as means \pm SD of three independent experiments.

Statistical evaluation. Data are reported as means \pm SD of three independent experiments. One-way ANOVA was used to assess statistical significance between the means. Significant differences were established at $\mathrm{P}<0.05$.

\section{Results}

ATRA activates the expression of p53. We previously cultured glioma C6 and U251 cell lines with or without ATRA for $24 \mathrm{~h}$, and the levels of mRNA and protein of Axin were examined by RT-PCR and western blotting, respectively. A marked increase in Axin was observed after ATRA treatment in the cell lines accompanied with significant growth inhibition of the glioma cells (18). These results indicates that ATRA affects tumor cell survival via activation of Axin. To ascertain whether p53 is involved in ATRA-induced cell cycle arrest and apoptosis in C6 cells, immunohistochemistry was employed to investigate the expression of p53 in ATRA-treated C6 cells. ATRA treatment increased the expression of p53 protein from $9.8 \pm 1.2$ to $19.6 \pm 2.2 \%$ (Fig. 1A). We then investigated the levels of p53 
A
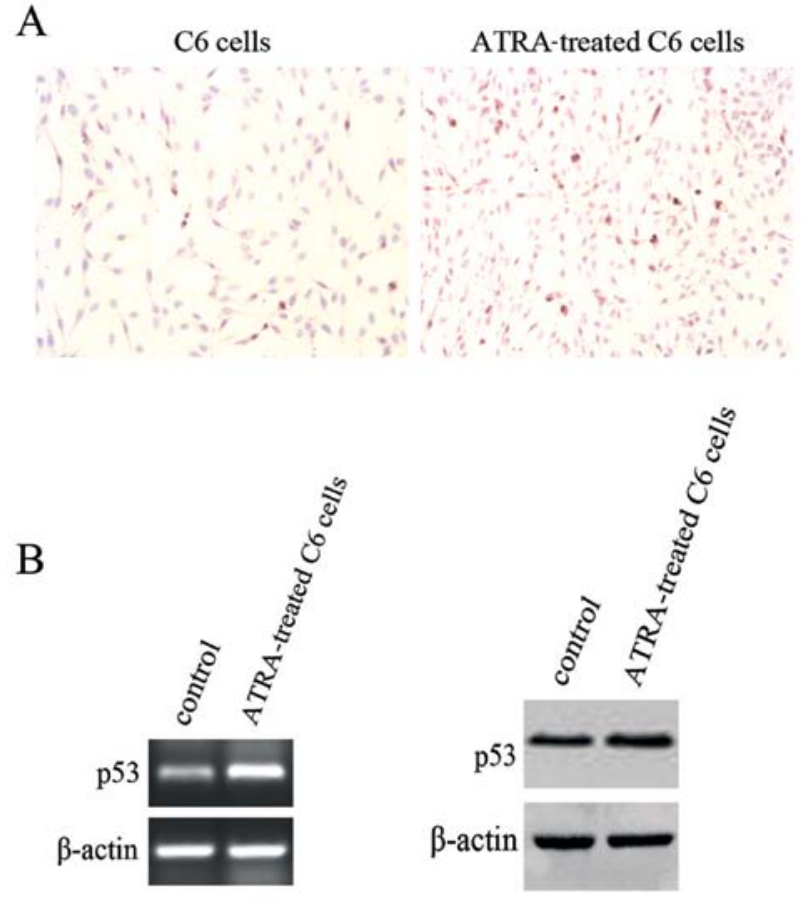

C

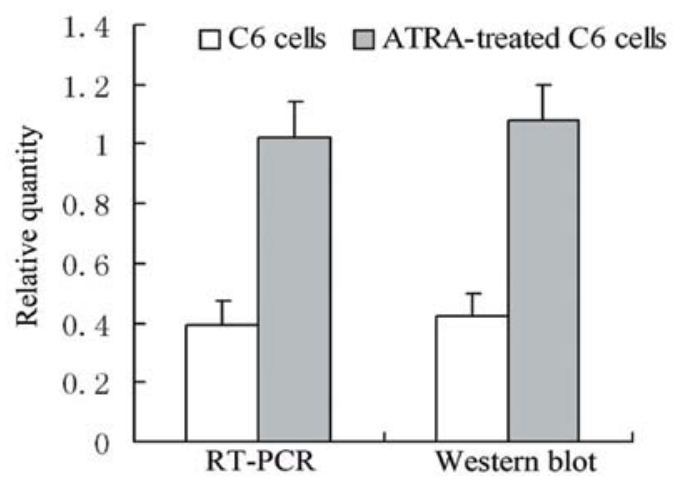

Figure 1. ATRA activates the expression of p53. C6 cells were cultured with or without ATRA for $24 \mathrm{~h}$. (A) Immunohistochemistry revealed that ATRA treatment increased the expression of $\mathrm{p} 53$ protein in glioma C6 cells $(\mathrm{n}=3$, $\mathrm{P}<0.05$ ). (B) RT-PCR and western blotting indicated that levels of $\mathrm{p} 53 \mathrm{mRNA}$ and protein were increased in the C6 cells following ATRA treatment $(n=3$, $\mathrm{P}<0.05)$. (C) Expression of mRNA and protein was normalized, respectively, to the quantity of $\beta$-actin.

mRNA and protein by RT-PCR and western blotting, respectively (Fig. 1B). The level of total p53 protein was significantly elevated in correspondence with an increase in its mRNA after ATRA treatment (Fig. 1C). These data suggest that the activation of p53 participates in ATRA-induced cell cycle arrest and apoptosis in C6 cells.

Overexpression of Axin activates the expression of $p 53$ and induces G1/S phase arrest and apoptosis. To identify whether overexpression of Axin induces the expression of p53, rat glioma C6 cells were transiently transfected with rat wild-type Axin. RT-PCR and western blotting were performed to detect the levels of p53 mRNA and protein (Fig. 2A and B). The levels of p53 mRNA and its protein in cells transfected with rAxin were increased compared to the levels in the cells
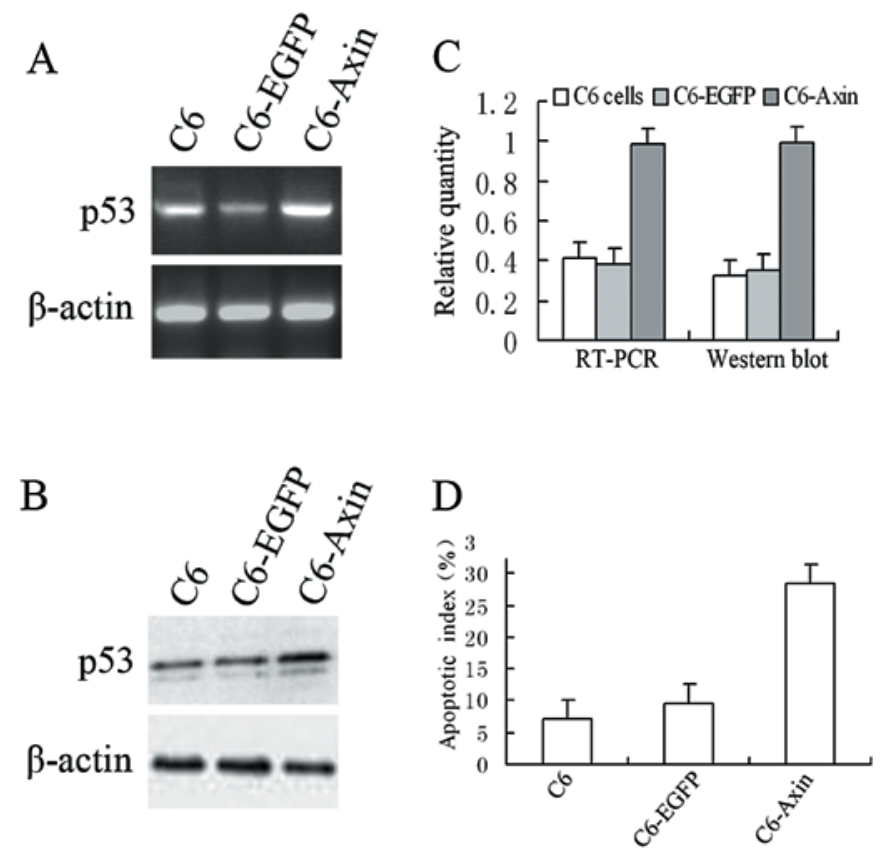

$\mathrm{E}$

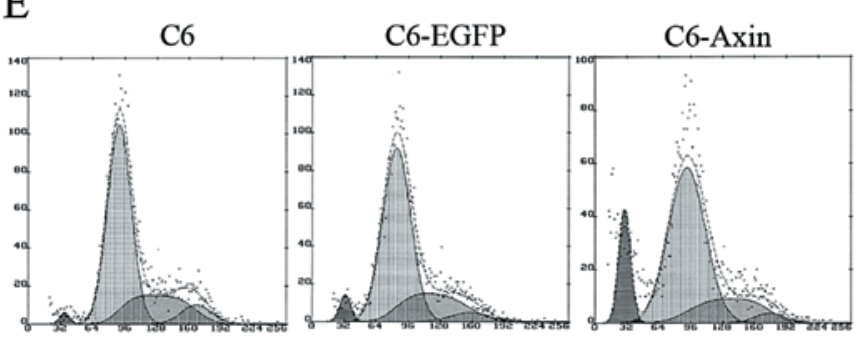

F

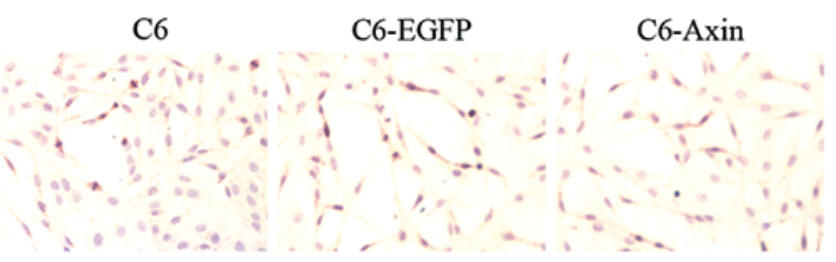

Figure 2. Overexpression of Axin activates the expression of p53 and induces G1/S phase arrest and apoptosis. C6 cells were transiently transfected with rat wild-type Axin (C6-Axin) or empty vector (C6-EGFP). Accompanied by the overexpression of Axin, the levels of p53 (A) mRNA and (B) protein in cells transfected with rAxin were increased compared to the levels in the cells transfected with the empty vector $(n=3, P<0.05)$. (C) Expression of mRNA and protein was normalized, respectively, to the quantity of $\beta$-actin. (E) Concerning C6 cells transfected with rAxin, flow cytometric analysis showed that the percentages of cells in pre-G1 and G1 phases were increased and the percentage of cells in the $\mathrm{S}$ phase was decreased compared to cells transiently transfected with the empty vector $(n=3, P<0.05)$. ( $D$ and $F)$ The apoptotic index of $\mathrm{C} 6$ transfected with Axin was significantly increased compared to that of C6 cells transfected with the empty vector and the C6 cells $(\mathrm{n}=5, \mathrm{P}<0.05)$.

transfected with the empty vector (Fig. 2C). These results confirmed that the overexpression of Axin was required for the activation of p53. To identify whether overexpression of Axin induces cell cycle arrest and apoptosis, flow cytometry (Fig. 2E) and TUNEL analysis (Fig. 2D and F) were used to determine the DNA content and apoptotic index, respectively. Flow cytometric analysis showed that the percentages of C6 cells transiently transfected with the empty vector in pre-G1, $\mathrm{G} 1$ and $\mathrm{S}$ phases were $4.8 \pm 1.4,63.6 \pm 2.8$ and $25.8 \pm 2.4 \%$, 

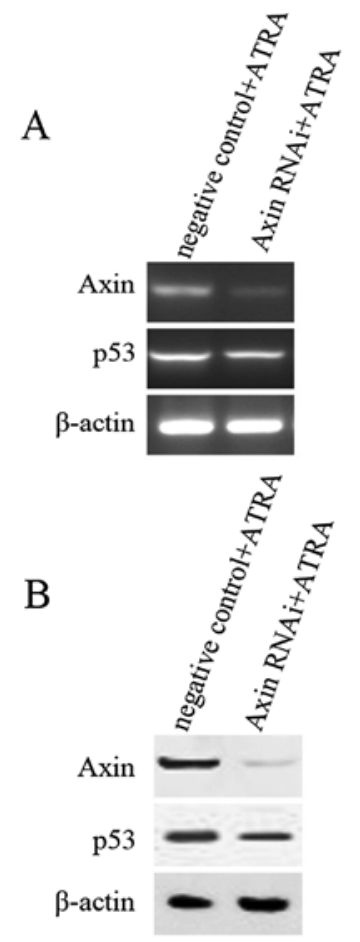
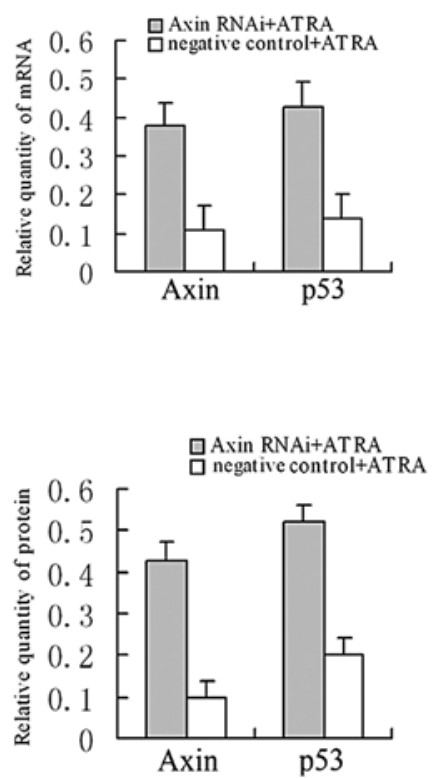

C

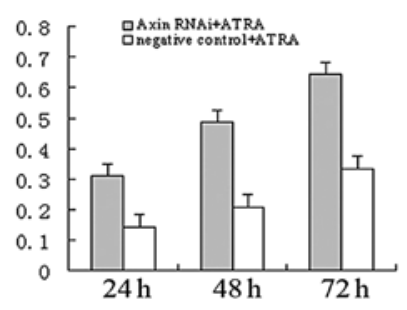

E

negative control+ATRA

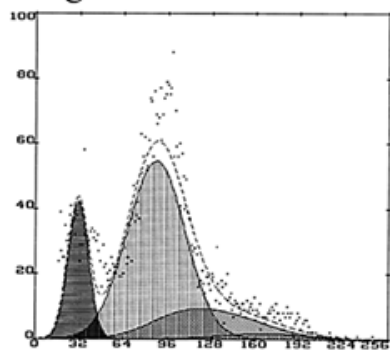

D

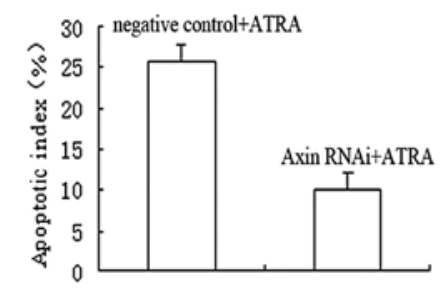

Axin-RNAi+ATRA

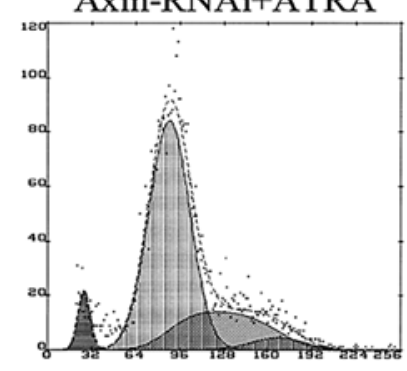

Figure 3. Axin RNAi decreases the expression of p53 and attenuates ATRA-induced G1/S phase arrest and apoptosis. C6 cells were transfected with siRNAs at a final concentration of $100 \mathrm{nM}$ using Lipofectamine 2000 according to the manufacturer's instructions. At $8 \mathrm{~h}$ post-transfection, cells were cultured with $2.5 \mu \mathrm{M}$ of ATRA for $24 \mathrm{~h}(\mathrm{n}=3, \mathrm{P}<0.05)$. (A) RT-PCR and (B) western blotting indicated that Axin mRNA was barely detected and protein expression was decreased significantly in cells transfected with the RNAi sequence against Axin following ATRA treatment for 24 h. As compared with the control, Axin-RNAi caused a decrease in p53 mRNA and its protein following ATRA treatment for $24 \mathrm{~h}(\mathrm{n}=3, \mathrm{P}<0.05)$. The expression of mRNA and protein was normalized, respectively, to the quantity of $\beta$-actin. (C) MTT assay indicated that the growth rate of the Axin RANi group was significantly decreased compared to the negative control group after transfection $(n=3, P<0.05)$. (D) TUNEL analysis revealed that the apoptotic index of cells with knockdown of Axin was significantly decreased compared to the negative control $(n=3, P<0.05)$. (E) Flow cytometric analysis revealed that Axin RNAi attenuated ATRA-induced G1/S phase arrest and apoptosis $(n=3, P<0.05)$.

respectively, while the percentages of C6 cells transfected with rAxin in pre-G1, G1 and $\mathrm{S}$ phases were $16.0 \pm 2.3,74.8 \pm 3.2$ and $11.6 \pm 1.7 \%$, respectively. The apoptotic index of C6 cells transfected with Axin (28.4 $\pm 3.3 \%)$ was significantly increased compared to the C6 cells transfected with the empty vector $(9.6 \pm 2.4 \%)$ and the C6 cells $(6.8 \pm 1.8 \%)$. These results revealed that overexpression of Axin inhibits cell proliferation and induces apoptotic cell death.

Axin RNAi attenuates ATRA-induced G1/S arrest and apoptosis accompanied by inhibition of the expression of p53. We performed RNA interference to further investigate the role of Axin in ATRA-induced cell cycle arrest and apoptotic cell death of C6 cells. Axin mRNA (Fig. 3A) was barely detected, and its protein expression (Fig. 3B) was downregulated significantly in cells transfected with the RNAi sequence against Axin following ATRA treatment. These data demonstrated that Axin-RNAi effectively silenced the ATRA-induced increase in Axin. Interestingly, Axin-RNAi caused only a moderate decrease in p53 mRNA and its protein following ATRA treatment (Fig. 3A and B). These data revealed that the inactivation of p53 is associated with the silencing of Axin and implies that there are other mechanisms participating in ATRA-induced activation of p53. We next examined the effect of silencing Axin expression on ATRA-mediated cell growth. As compared with the control, the percentages of cells in the pre-G1 and G1 phases of the cell cycle were decreased
$(12.6 \pm 2.3$ and $14.9 \pm 2.8 \%$, respectively), while the percentage

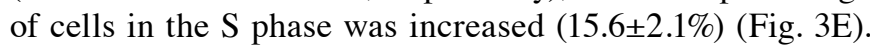
Forty-eight hours after transfection, the viability of the Axin RNAi-transfected C6 cells decreased by $\sim 60 \%$ compared with the control according to the MTT assay (Fig. 3C). TUNEL analysis revealed that the apoptotic index of cells with knockdown of Axin significantly decreased compared to the negative control (from 25.8 \pm 2.5 to $10.1 \pm 2.3 \%$ ) (Fig. 3D). These data reinforced the finding that Axin is an efficient regulator of ATRA-induced G1/S phase arrest and apoptosis, and silencing of its expression compromised the susceptibility to the effects of ATRA.

\section{Discussion}

The aggressiveness of cancer cells results from their proliferative advantage over their normal counterparts and from their inability to undergo apoptosis. Consistent with previous research (21-24), our results demonstrated that ATRA induced cell cycle arrest as well as apoptosis in glioma cells. The present study for the first time demonstrated that ATRA-mediated cell cycle arrest and apoptosis were associated with the activation of p53 resulting from the activation of Axin and this activation may be a general mechanism contributing to ATRA-inhibited tumor cell growth.

The tumor suppressor protein p53 is a nuclear phosphoprotein that can potently regulate the growth of mammalian 
cells (25). Activation of p53 results in altered transcription of a wide variety of genes that are involved in many aspects of cell metabolism, cell cycle regulation and apoptosis. Moreover, the introduction of a wild-type p53 expression vector into tumor cells including glioma cells suggests its ability to halt the cell cycle, trigger apoptosis, and prevent undue cell proliferation (26-29). ATRA regulation of the fate of cells by $\mathrm{p} 53$ has been reported (30). These studies revealed that ATRA induces cell cycle arrest and apoptosis by regulating the transcriptional activity of p53 and/or stability of its protein. However, whether p53 participates in ATRA-mediated growth control in glioma cells remains unclear. Current evidence showed that G1/S arrest and apoptosis induced by ATRA were associated with the activity of p53 in the C6 glioma cell line.

Previous research revealed that Axin participates in ATRA-mediated differentiation of embryonic carcinoma cells (17) and overexpression of Axin causes apoptotic cell death in transgenic mice and certain cell lines $(12,13)$. Recently, our group found that the activation of Axin was required for ATRA-inhibited cellular proliferation in glioma cell lines (18). Here, we found that overexpression of Axin by transiently transfecting C6 cells with rAxin inhibited the G1/S phase progression in addition to inducing apoptotic cell death. These results were further confirmed by Axin gene silencing. Herein, we propose that the activation of Axin, as well as the activation of $\mathrm{p} 53$, inhibited cell growth by cell cycle arrest and apoptotic cell death in the C6 glioma cell line following ATRA treatment.

Axin has been reported to stimulate p53 and p53-dependent transcriptional activity by the formation of a complex with p53 (19). This raised an intriguing possibility that ATRA-activated expression of Axin may inhibit tumor cell growth and activate the expression of p53. In the present study, we provide evidence that ATRA increases the expression of p53 through the activation of Axin. As mentioned above, p53, widely expressed in normal adult tissues, tumors, and embryos, is ubiquitously involved in growth arrest and/or apoptosis via a multitude of molecular pathways involving transactivation of target genes and direct signaling events (31-33). Base on these results, it is legitimate to suggest that Axin is an important regulator of ATRA-induce growth arrest and apoptosis of C6 glioma cells by mediating the p53-dependent cell death pathway, and this may be a general mechanism contributing to ATRA-mediated cell cycle arrest and apoptotic cell death.

\section{Acknowledgements}

The present study was supported by grants from the State Key Laboratory of Cancer Biology, China (CBSKL 2005004) and the Science and Technology Department of Shaanxi Province, China (2011JM4005). We thank Dr Zhizhong Wang at the Department of Epidemiology of The Fourth Military Medical University for assistance with the statistical analysis.

\section{References}

1. Zauli G, Visani G, Bassini A, et al: Nuclear translocation of protein kinase C-alpha and -beta isoforms in HL-60 cells induced to differentiate along the granulocytic lineage by all-trans retinoic acid. Br J Haematol 93: 542-550, 1996.
2. Yung WK, Lotan R and Lee P: Modulation of growth and epidermal growth factor receptor activity by retinoic acid in human glioma cells. Cancer Res 49: 1014-1019, 1989.

3. Idres N, Benoît G, Flexor MA, Lanotte M and Chabot GG: Granulocytic differentiation of human NB4 promyelocytic leukemia cells induced by all-trans retinoic acid metabolites. Cancer Res 6: 700-705, 2001.

4. Launay S, Gianni M, Diomede L, et al: Enhancement of ATRAinduced cell differentiation by inhibition of calcium accumulation into the endoplasmic reticulum: cross-talk between $\operatorname{RAR} \alpha$ and calcium-dependent signaling. Blood 101: 3220-3228, 2003.

5. Chambon P: A decade of molecular biology of retinoic acid receptors. FASEB J 10: 940-954, 1996.

6. Mrass P, Rendl M, Mildner M, et al: Retinoic acid increases the expression of p53 and proapoptotic caspases and sensitizes keratinocytes to apoptosis: a possible explanation for tumor preventive action of retinoids. Cancer Res 64: 6542-6548, 2004.

7. Um SJ, Kim EJ, Hwang ES, et al: Antiproliferative effects of retinoic acid/interferon in cervical carcinoma cell lines: cooperative growth suppression of IRF-1 and p53. Int J Cancer 85: 416-423, 2000 .

8. Zheng A, Mäntymaa P, Säily M, et al: p53 pathway in apoptosis induced by all-trans-retinoic acid in acute myeloblastic leukaemia cells. Acta Haematol 103: 135-143, 2000.

9. Ronca F, Yee KS and Yu VC: Retinoic acid confers resistance to p53-dependent apoptosis in SH-SY5Y neuroblastoma cells by modulating nuclear import of p53. J Biol Chem 274: 18128-18134, 1999.

10. Curtin JC, Dragnev KH, Sekula D, Christie AJ, Dmitrovsky E and Spinella MJ: Retinoic acid activates p53 in human embryonal carcinoma through retinoid receptor-dependent stimulation of p53 transactivation function. Oncogene 20: 2559-2569, 2001.

11. Zeng L, Fagotto F, Zhang T, et al: The mouse Fused locus encodes Axin, an inhibitor of the Wnt signaling pathway that regulates embryonic axis formation. Cell 90: 181-192, 1997.

12. Satoh S, Daigo Y, Furukawa Y, et al: AXIN1 mutations in hepatocellular carcinomas, and growth suppression in cancer cells by virus-mediated transfer of AXIN1. Nat Genet 24: 245-250, 2000.

13. Hsu W, Shakyo R and Costantini F: Impaired mammary gland and lymphoid development caused by inducible expression of Axin in transgenic mice. J Cell Biol 155: 1055-1064, 2001.

14. Peifer $M$ and Polakis P: Wnt signaling in oncogenesis and embryogenesis - a look outside the nucleus. Science 287: 1606-1609, 2000.

15. Luo W, Ng WW, Jin LH, Ye Z, Han J and Lin SC: Axin utilizes distinct regions for competitive MEKK1 and MEKK4 binding and JNK activation. J Biol Chem 278: 37451-37458, 2003.

16. Liu W, Rui H, Wang J, et al: Axin is a scaffold protein in TGF- $\beta$ signaling that promotes degradation of Smad7 by Arkadia. EMBO J 25: 1646-1658, 2006.

17. Lyu J, Costantini F, Jho EH and Joo CK: Ectopic expression of Axin blocks neuronal differentiation of embryonic carcinoma P19 cells. J Biol Chem 278: 13487-13495, 2003.

18. Lu J, Zhang F, Zhao D, et al: ATRA-inhibited proliferation in glioma cells is associated with subcellular redistribution of $\beta$-catenin via up-regulation of Axin. J Neurooncol 87: 271-277, 2008.

19. Rui Y, Xu Z, Lin S, et al: Axin stimulates p53 functions by activation of HIPK2 kinase through multimeric complex formation. EMBO J 23: 4583-4594, 2004.

20. Li QX, Wang X, Wu X, et al: Daxx cooperates with the Axin/HIPK2/px53 complex to induce cell death. Cancer Res 67: 66-74, 2007.

21. Karmakar S, Banik NL and Ray SK: Combination of all-trans retinoic acid and paclitaxel-induced differentiation and apoptosis in human glioblastoma U87MG xenografts in nude mice. Cancer 112: 596-607, 2008.

22. Papi A, Bartolini G, Ammar K, Guerra F, Ferreri AM, Rocchi P and Orlandi M: Inhibitory effects of retinoic acid and IIF on growth, migration and invasiveness in the U87MG human glioblastoma cell line. Oncol Rep 18: 1015-1021, 2007.

23. Karmakar S, Banik NL, Patel SJ and Ray SK: Combination of all-trans retinoic acid and taxol regressed glioblastoma T98G xenografts in nude mice. Apoptosis 12: 2077-2087, 2007.

24. Zhang R, Banik NL and Ray SK: Combination of all-trans retinoic acid and interferon-gamma suppressed PI3K/Akt survival pathway in glioblastoma T98G cells whereas NF-kappaB survival signaling in glioblastoma U87MG cells for induction of apoptosis. Neurochem Res 32: 2194-2202, 2007.

25. Vogelstein B and Kinzler KW: p53 function and dysfunction. Cell 70: 523-526, 1992. 
26. Levine AJ: p53, the cellular gatekeeper for growth and division. Cell 88: 323-331, 1997.

27. Timiryasova TM, Chen B, Haghighat P and Fodor I: Vaccinia virus-mediated expression of wild-type p53 suppresses glioma cell growth and induces apoptosis. Int J Oncol 14: 845-854, 1999.

28. Cirielli C and Inyaku K: Adenovirus-mediated wild-type p53 expression induces apoptosis and suppresses tumorigenesis of experimental intracranial human malignant glioma. J Neurooncol 43: 99-108, 1999.

29. Merzak A, Raynal S, Rogers JP, Lawrence D and Pilkington GJ: Human wild type p53 inhibits cell proliferation and elicits dramatic morphological changes in human glioma cell lines in vitro. J Neuro Sci 127: 125-133, 1994.

30. Shin DM, Xu XC, Lippman SM, et al: Accumulation of $\mathrm{p} 53$ protein and retinoic acid receptor $\beta$ in retinoid chemoprevention. Clin Cancer Res 3: 875-880, 1997.
31. Li PF, Dietz R and von Harsdorf R: p53 regulates mitochondrial membrane potential through reactive oxygen species and induces cytochrome $\mathrm{c}$ independent apoptosis blocked by Bcl-2. EMBO J 18: 6027-6036, 1999.

32. Lee SW, Fang L, Igarashi M, Ouchi T, Lu KP and Aaronson SA: Sustained activation of Ras/Raf/mitogen-activated protein kinase cascade by the tumor suppressor p53. Proc Natl Acad Sci USA 97: 8302-8305, 2000.

33. Yamaguchi A, Tamatani M, Matsuzaki H, et al: Akt activation protects hippocampal neurons from apoptosis by inhibiting transcriptional activity of p53. J Biol Chem 271: 31929-31936, 1996. 